\title{
Diagnosis and management of systemic Wegener's granulomatosis presenting with anterior ocular inflammatory disease
}

\author{
Stephen J Charles, Paul A R Meyer, Peter G Watson
}

\begin{abstract}
The ocular and systemic features of 10 patients whose Wegener's granulomatosis presented with corneoscleral inflammatory disease are described. Marginal corneal infiltrates were seen in all patients with anterior scleritis and were a valuable sign of disease activity. Nine out of 10 patients had symptoms of systemic vasculitis on presentation; seven had renal impairment; three had chest $x$-ray abnormalities. Autoantibodies against neutrophil cytoplasmic determinants (ANCA) were present in all cases. In seven patients the scleritis responded well to pulsed immunosuppressive therapy followed by long term oral steroids and cyclophosphamide. Oral steroid therapy alone failed to control severe disease. Corneoscleral disease was not a cause of visual loss. It is important to realise that inflammatory corneoscleral disease may be the presenting feature of a severe systemic vasculitis.
\end{abstract}

Wegener's granulomatosis (WG) is a multifocal necrotising vasculitis which most commonly affects the lungs, upper respiratory tract, and kidneys. ${ }^{12}$ In consequence patients may present with haemoptysis from cavitating areas of pulmonary consolidation, sinusitis and serous otitis media, or renal failure resulting from chronic glomerulonephritis. Untreated, the disease used to be rapidly fatal, usually as a result of renal failure. $^{3}$

The eye is involved in between $28 \%{ }^{4}$ and $58 \%{ }^{2}$ of cases, and patients often present with the ocular manifestations. There may be orbital invasion by paranasal sinus granulomata, producing painful proptosis with ophthalmoplegia and optic nerve compression, or a focal vasculitis $^{67}$ causing scleritis and peripheral corneal disease. In the past ocular involvement caused severe visual loss regardless of treatment. ${ }^{8}$

The affected tissues show a necrotising vasculitis with granuloma formation,' and leucocyte fragments have been demonstrated in the lumina of the inflamed vessels by electron microscopy. ${ }^{9}$ The characterisation of the disease has recently been facilitated by the detection of autoantibodies against neutrophil cytoplasmic determinants (ANCA) in patients with systemic vasculitis, especially WG and microvascular polyarteritis (MPA). ${ }^{10-12}$ Two types of autoantibody have recently been identified: c-ANCA active against a $29 \mathrm{kD}$ protease ${ }^{13}$ and p-ANCA directed against myeloperoxidase. ${ }^{14}$ The former appears to be specific for WG, while p-ANCA has been found in other forms of vasculitis such as MPA. ${ }^{15}$

The treatment of choice in WG has been a combination of systemic corticosteroids and a lymphocytotoxic agent, of which cyclophosphamide is the most effective. ${ }^{216}$ Pulsed intravenous corticosteroids and cyclophosphamide, ${ }^{17}$ and plasma exchange, ${ }^{18}$ have also been used to control the acute phase of the disease.

In this study we describe the ocular and systemic features of a series of patients whose WG presented with anterior ocular disease. We also consider the consequences for ocular morbidity when treatment is directed against the underlying systemic disease.

Table 1 Results of investigations at presentation with eye disease

Department of
Ophthalmology,
Addenbrooke's Hospital,
Cambridge CB2 2QQ
S J Charles
P A R Meyer
P G Watson
Correspondence to:
Mr S J Charles, FRCS:
Accepted for publication
26 October 1990

\begin{tabular}{|c|c|c|c|c|c|c|c|c|c|c|}
\hline \multirow[b]{3}{*}{ Investigation } & \multirow{2}{*}{\multicolumn{3}{|c|}{ Scleritis without necrosis }} & \multicolumn{7}{|c|}{ Scleritis with necrosis } \\
\hline & & & & \multicolumn{3}{|c|}{ without gutter } & \multicolumn{4}{|c|}{ with gutter } \\
\hline & 1 & 2 & 3 & 4 & 5 & 6 & 7 & 8 & 9 & 10 \\
\hline \multirow{5}{*}{ 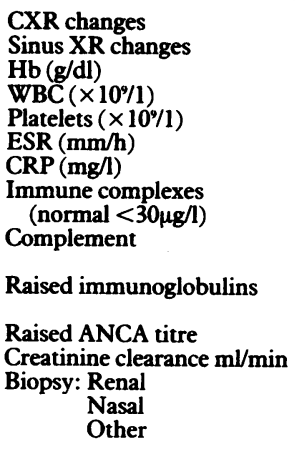 } & $\begin{array}{l}- \\
+ \\
13 \cdot 8 \\
5 \cdot 9 \\
432 \\
19^{\star} \\
\mathrm{ND}\end{array}$ & $\begin{array}{l}- \\
- \\
12 \cdot 1 \\
10 \cdot 8 \\
534 \\
123 \\
123\end{array}$ & $\begin{array}{l}- \\
+ \\
10 \cdot 3 \\
13 \cdot 5 \\
847 \\
129 \\
192\end{array}$ & $\begin{array}{l}+ \\
- \\
11 \cdot 6 \\
14 \cdot 8 \\
495 \\
83 \\
109\end{array}$ & $\begin{array}{c}- \\
+ \\
13 \cdot 2 \\
7 \cdot 9 \\
263 \\
32^{\star} \\
9 \star\end{array}$ & $\begin{array}{l}- \\
+ \\
12 \cdot 4 \\
12 \cdot 0 \\
386 \\
114 \\
97\end{array}$ & $\begin{array}{l}+ \\
+ \\
9 \cdot 4 \\
11 \cdot 8 \\
832 \\
121 \\
138\end{array}$ & $\begin{array}{l}+ \\
+ \\
11 \cdot 3 \\
11 \cdot 2 \\
814 \\
89 \\
57\end{array}$ & $\begin{array}{c}- \\
+\quad 8 \cdot 0 \\
10 \cdot 8 \\
651 \\
>140 \\
284\end{array}$ & $\begin{array}{c}- \\
- \\
15 \cdot 3 \\
8 \cdot 9 \\
243 \\
35 \\
16\end{array}$ \\
\hline & $\begin{array}{l}<30 \\
\mathrm{~N}\end{array}$ & $\begin{array}{c}10 \\
\text { Raised } \\
\text { C3C4 }\end{array}$ & $\mathrm{N}^{28}$ & $N^{4}$ & $\begin{array}{l}\mathbf{N D} \\
\mathbf{N}\end{array}$ & $\begin{array}{c}40 \\
\text { Raised } \\
\text { C4 }\end{array}$ & $\begin{array}{c}72 \\
\text { Raised } \\
\text { C3C4 }\end{array}$ & $\begin{array}{l}33 \\
\mathbf{N}\end{array}$ & $\begin{array}{l}\text { ND } \\
\mathbf{N}\end{array}$ & $\begin{array}{l}\text { ND } \\
\text { ND }\end{array}$ \\
\hline & $\mathbf{N}$ & IgA & IgG & $\mathbf{N}$ & $\mathbf{N}$ & IgG & IgG & IgA & ND & $\mathbf{N}$ \\
\hline & $\begin{array}{l}+ \\
+ \\
+\end{array}$ & $\begin{array}{l}+ \\
+84.5 \\
+\end{array}$ & $+_{62.5}$ & + & $\ddagger_{71 \cdot 3}$ & + & + & $\begin{array}{l}+ \\
20 \cdot 5\end{array}$ & +3.84 & $\stackrel{\ddagger}{126}$ \\
\hline & & & + & + & + & + & + & + & + & + \\
\hline
\end{tabular}

$\star$ On steroid therapy. $\mathrm{ND}=$ not done. $\mathrm{N}=$ normal. $\uparrow$ ANCA testing not yet available when patient 1 initially presented, but ANCA was elevated when tested later. $¥$ Became raised. 


\section{Patients and methods}

Between 1986 and 1989 ten patients (six females and four males) were seen in Addenbrooke's Hospital with scleritis complicating WG. All presented with scleritis, with or without corneal guttering, and had features compatible with WG in nasal mucosal or renal biopsies, together with increased ANCA.

Each patient underwent full medical examination, and their investigations are listed in Table 1. The ANCA were measured by a solid phase radioimmunoassay (RIA); test sera were quantified as a percentage of the binding of a reference strong positive serum (normal range $<16 \%$ ). Indirect immunofluorescence (IIF) was performed to distinguish granular cytoplasmic staining (c-ANCA) from a perinuclear staining pattern (p-ANCA).

\section{Clinical findings}

The patients were divided into two groups according to the absence of presence of scleral necrosis. The age at onset of disease ranged from 38-65 years (mean 56.6 years).

\section{SCLERITIS WITHOUT SCLERAL NECROSIS}

\section{Systemic disease}

The first patient in this group appeared to suffer from scleritis alone, but developed renal failure four years later. Two other patients suffered from arthralgia at the time of their presentation; one also complained of epistaxis. Both were found to have renal disease. None had any evidence of lung disease.

Renal biopsies in two patients demonstrated respectively mesangioproliferative glomerulonephritis and acute glomerular necrosis. In the third patient nasal mucosal biopsy showed necrotising vasculitis affecting small arteries and venules, with granuloma formation. The ANCA titre (RIA) was raised in all three cases, and IIF confirmed granular cytoplasmic staining in two cases.

\section{Ocular disease}

All patients had anterior scleritis (Fig 1), in which different sectors became involved during the course of the disease; a mild anterior uveitis was also present in one.

Limbal subepithelial infiltrates were seen in two cases, but thinning was not present. In one case areas of corneal epithelial disturbance were seen.

Case history: patient 2: scleritis, limbal ischaemia and proteinuria

A 51-year-old woman with a two-year history of recurrent episcleritis presented with right anterior scleritis and marginal corneal subepithelial infiltrates. She also complained of a painful swollen foot. The erythrocyte sedimentation rate (ESR) was $123 \mathrm{~mm} / \mathrm{h}$ and despite normal urea and electrolytes there was heavy proteinuria and reduced creatinine clearance. Chest and sinus $x$-rays were normal. The ANCA

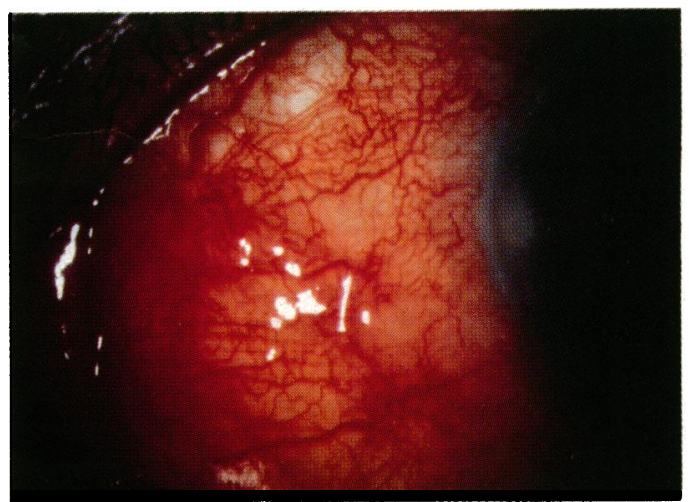

Figure 1 Anterior scleritis without scleral necrosis (patient 3) may be the presenting feature of WG. Scleral oedema is accompanied by episcleral venous congestion.

titre was not raised and the eyes settled without treatment.

Four weeks later the ANCA titre rose $(\mathrm{RIA}=$ $44 \%$, and renal biopsy showed mesangial proliferation compatible with, but not diagnostic of, a systemic vasculitis such as WG or MPA. No treatment was instituted.

Four months later she developed bilateral multifocal marginal corneal epithelial defects (Fig 2) and granular subepithelial deposits. Herpetic infection was suspected, but no virus could be isolated, and there was no response to topical antiviral agents. Haemoglobin video imaging showed severe limbal hypoperfusion, and the epithelial defects were therefore attributed to limbal ischaemia. Her systemic vasculitis was controlled by cyclophosphamide and prednisolone, and, after two weeks, perfusion of the limbal arcades had returned to normal and the epithelial defects had disappeared. Leucopenia and alopecia prompted reduction of the cyclophosphamide dose. She has had episodes of arthralgia but is currently controlled on cyclophosphamide $(1.5 \mathrm{mg} / \mathrm{kg})$ and prednisolone $10 \mathrm{mg}$ daily.

\section{SCLERITIS WITH SCLERAL NECROSIS}

\section{Systemic disease}

Seven patients (four males, three females) presented with necrotising scleritis. All had symptoms of systemic disease; arthralgia (3), cough and breathlessness (2), pain over the

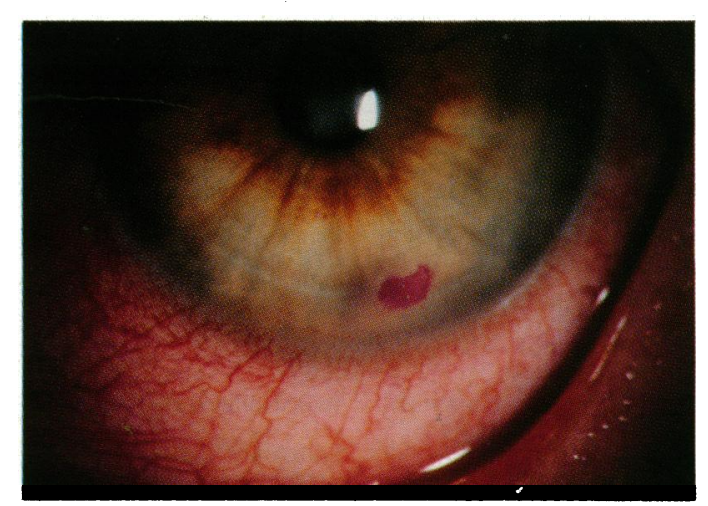

Figure 2 Unusual marginal corneal epithelial defect demonstrated by rose-Bengal stain (patient 2). Haemoglobin videoimaging showed hypoperfusion of the adjacent limbal arcades. The lesion was not affected by antiviral therapy but resolved after immunosuppression. 


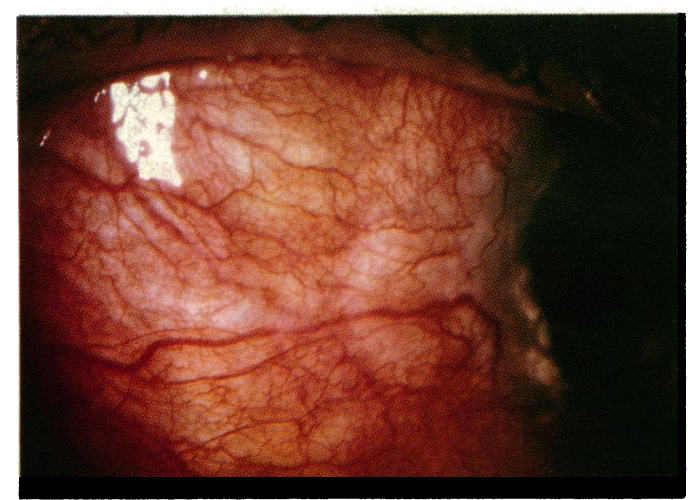

Figure 3 Anterior scleral necrosis with conjunctival cover (Patient 4). Note yellow colour of necrotic sclera and the adjacent marginal corneal infiltrates, which are multifocal, grey and have diffuse edges.

maxillary sinuses (6), epistaxis (6), and deafness (3).

Sinus $x$-rays revealed mucosal thickening or sinus opacification in five patients. Three cases had chest $x$-ray evidence of pulmonary consolidation, two with cavitation (Fig 6). Creatinine clearance indicated renal impairment in five patients.

Nasal mucosal biopsy provided histological confirmation of vasculitis in six patients and showed granuloma formation in five. Conjunctival biopsy (prior to referral) had shown vasculitis in a further case. The ANCA titre was also raised in all these patients. However, one had a normal titre at presentation, which was raised 18 months later. IIF could be demon-

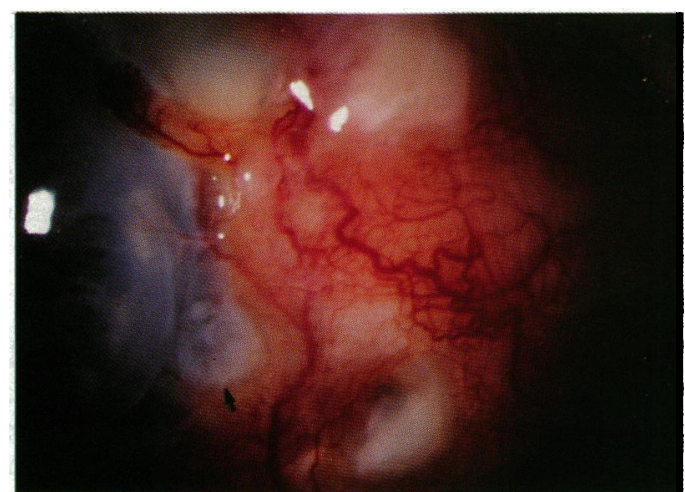

Figure 5A Corneal guttering central to areas of scleral necrosis with loss of conjunctival cover (patient 7). Note collagen plug in corneal gutter (arrowed) and necrotic tissue emerging from conjunctival defects.

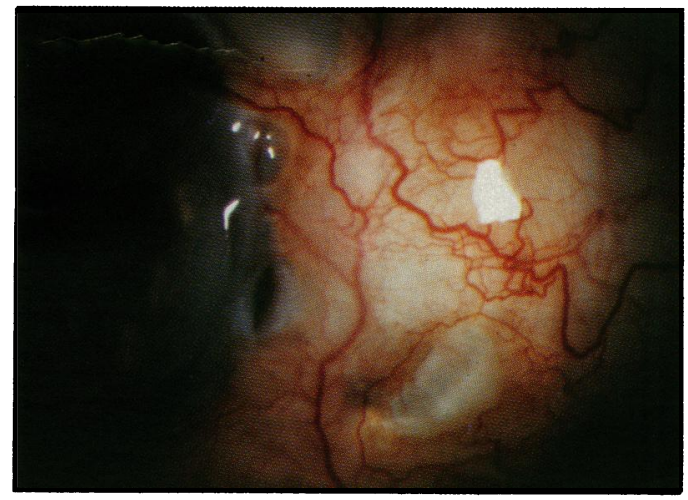

Figure 5B Same patient one week later, after two pulses of intravenous cyclophosphamide and methylprednisolone. The corneal plug from the nasal gutter has been lost, revealing a limbal perforation.

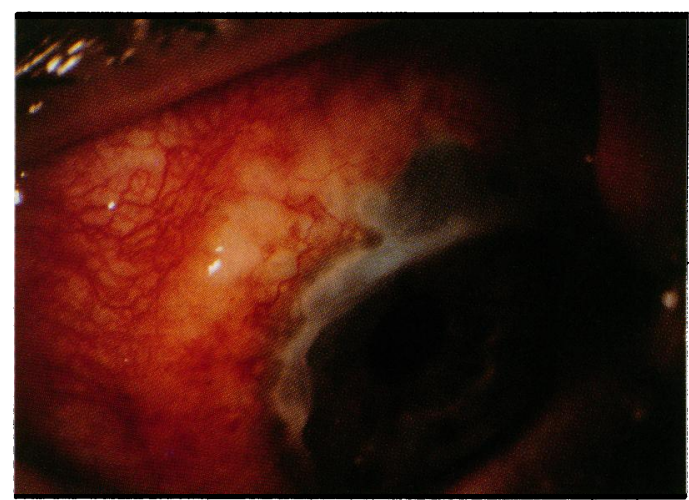

Figure 4 Anterior scleral necrosis with loss of conjunctival cover. The scleral necrosis is confluent with a circumferential corneal melt (patient 8).

strated in six cases, and in all of these c-ANCA was confirmed.

\section{Ocular disease}

Scleritis. Scleral necrosis appeared to arise at a single focus, which evolved either by enlargement or by the development of satellite lesions (Fig 5A). Superior sclera was involved in four subjects, inferior in one and nasal in three. Ultrasound demonstrated spread to postequatorial sclera in two patients. Necrotic sclera appeared yellow when viewed with low intensity, diffuse light (Fig 3). The overlying conjunctiva remained intact in two patients (Fig 3 ) but had become lost in five by the time of presentation (Figs 4, 5A, 7A).

Subconjunctival haemorrhage was present in five patients.

Limbal changes. The scleral necrosis transgressed the limbus in three patients (Fig 4) and evidence of limbal vascular remodelling was found in the remainder.

Corneal infiltrates. Multifocal stromal infiltrates below Bowman's membrane extended centrally from the limbus in every patient (Fig 3). Disease activity was accompanied by enlargement of the infiltrates and loss of definition of their borders. Conversely, during periods of remission they became smaller and more discrete (Fig 7d).

Corneal guttering was present in four patients. Gutters arose only central to areas of scleral necrosis (Fig 5A). Circumferential progression was characterised by stromal oedema at the

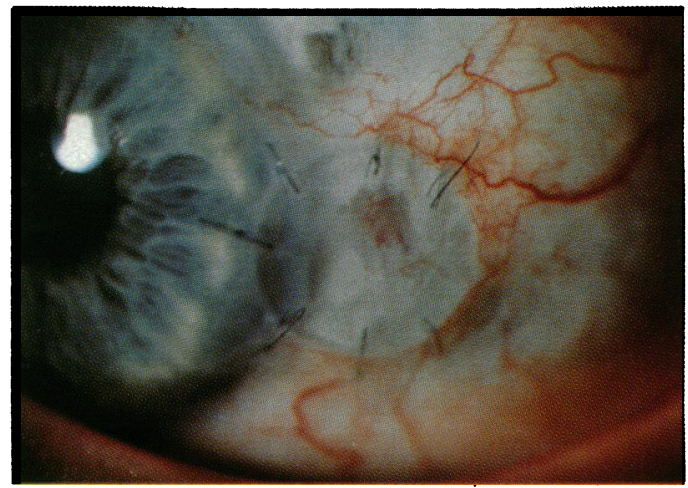

Figure 5C Lamellar corneoscleral graft performed after medical control of disease activity. 
leading edge. The central limit of the gutter was shelving in three cases and steep in one. Limbal perforation occurred in one patient (Fig 5B) but anterior chamber depth was maintained, allowing corrective corneoscleral lamellar patch grafting to be delayed until disease activity had been fully controlled (Fig 5C).

Uveitis. A few cells and minimal flare were present in four cases.

Case history: patient four: nodular scleritis with cavitating lung lesions

A 39-year-old woman, with a past history of polyarthralgia, developed left anterior scleritis. This initially responded to high dose oral steroids, but then relapsed accompanied by a cough, a sore throat and malaise. She first attended our unit with visual acuities of $6 / 5$ OD and 6/36 OS, left nodular scleritis with disruption of the adjacent limbal arcade, and superficial marginal stromal corneal infiltrates (Fig 3).

Chest $x$-ray revealed multiple cavitating areas of pulmonary consolidation (Fig 6), but sinus $x$ rays were normal. The ESR, c-reactive protein (CRP), and ANCA titre were raised, but renal function was normal. Though ear, nose, and throat examination gave normal findings, nasal mucosal biopsy showed active vasculitis with a giant cell infiltrate, granuloma formation, and necrosis diagnostic of WG.

After three pulses of intravenous methylprednisolone and two of cyclophosphamide her scleral nodule diminished. Despite daily oral prednisolone $(20 \mathrm{mg}$ ) and cyclophosphamide $(1.5 \mathrm{mg} / \mathrm{kg})$, scleritis recurred in a different site one month later. This responded to two further pulses of methylprednisolone and cyclophosphamide. Maintenance cyclophosphamide was increased, but after six months was replaced

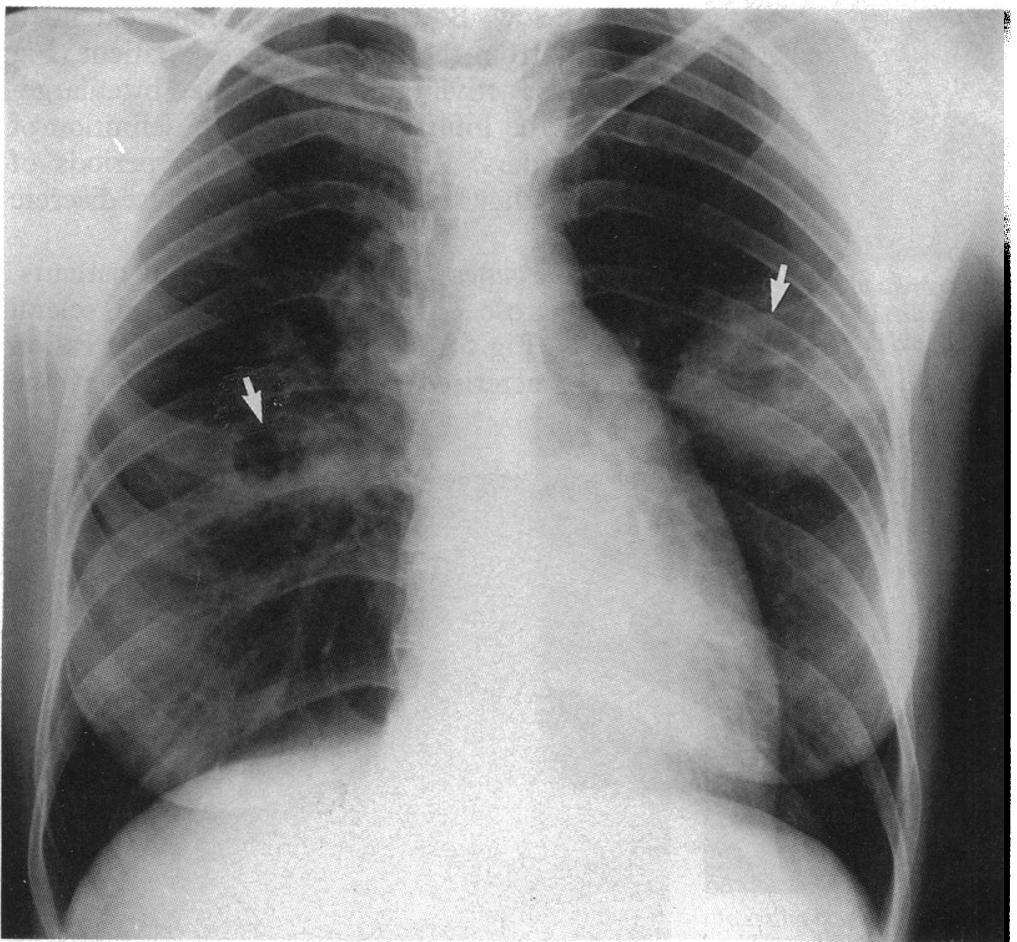

Figure 6 Chest $\mathrm{x}$-ray (patient 4) showing cavitation within areas of pulmonary consolidation (arrowed), typical of WG. by azathioprine. Her scleritis relapsed on the new regimen, now involving postequatorial sclera with severe posterior uveitis. She also developed a pericardial effusion. Cyclophosphamide was restored and the scleritis is now controlled, but visual acuity in the affected eye is reduced to counting fingers $(C F)$ by a macular hole complicating chronic cystoid macular oedema secondary to posterior uveitis.

Case history: patient 8. Scleral necrosis, deafness, pulmonary cavitation, and renal failure

A 64-year-old man presented with a two-month history of arthralgia affecting his knees and elbows, nasal obstruction, and epistaxis, then soreness of both eyes and unilateral deafness. Visual acuities were 6/6 OD and 6/9 OS. There was bilateral superior scleral necrosis, with necrosis of the overlying conjunctiva, widespread pericapillary haemorrhages, and damage to the limbal vascular arcades (Fig 4). Marginal corneal infiltrates were accompanied by early corneal gutter formation and mild anterior uveitis.

The ESR and CRP were raised and there were microscopic haematuria, proteinuria, and reduced creatinine clearance $(20.5 \mathrm{ml} / \mathrm{min})$. Chest $x$-ray showed a cavitating pulmonary lesion, and sinus $x$-rays showed antral mucosal thickening. The ANCA titre was found to be raised. Nasal mucosal biopsy showed features suggestive of WG and renal biopsy severe necrotising glomerulonephritis. The patient was treated with pulsed intravenous methylprednisolone and cyclophosphamide. Despite an improvement in his scleritis, his creatinine clearance continued to fall and plasma exchange was started. This reduced circulating ANCA and improved renal function. His disease is currently controlled by prednisolone $10 \mathrm{mg}$ and cyclophosphamide $40 \mathrm{mg}$ daily, and both eyes have retained visual acuities of $6 / 6$.

\section{Treatment}

All patients were admitted to hospital for investigation and clinical evaluation.

Non-steroidal anti-inflammatory agents had been used prior to admission in six patients but were never successful.

Oral steroids were used in all patients but alone never controlled acute disease. In two patients (without scleral necrosis) control was achieved by additional oral cyclophosphamide.

Pulsed immunosuppressive therapy (intravenous methylprednisolone and cyclophosphamide ${ }^{19}$ ) was used in the seven patients, six with scleral necrosis, who had not responded to oral steroids and in whom scleritis was progressing rapidly. Intravenous injections of methylprednisolone $(0.5-1.0 \mathrm{~g})$ were repeated at three-day intervals until the scleritis had subsided: no more than four injections were ever required. Cyclophosphamide $(0.5 \mathrm{~g})$ usually accompanied the first two pulses of methylprednisolone. Oral prednisolone $(15-20 \mathrm{mg}$ ) was started with the first pulse, and cyclophosphamide $(2 \mathrm{mg} / \mathrm{kg}$ ) was added when its intravenous administration ceased. This treatment acheived control of scleritis in all patients in whom it was used. 


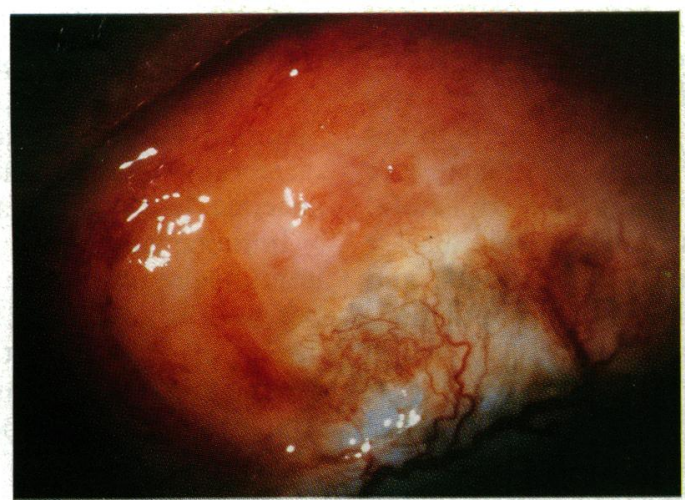

Figure 7A Necrotising scleral granuloma with loss of conjunctival cover (patient 5).

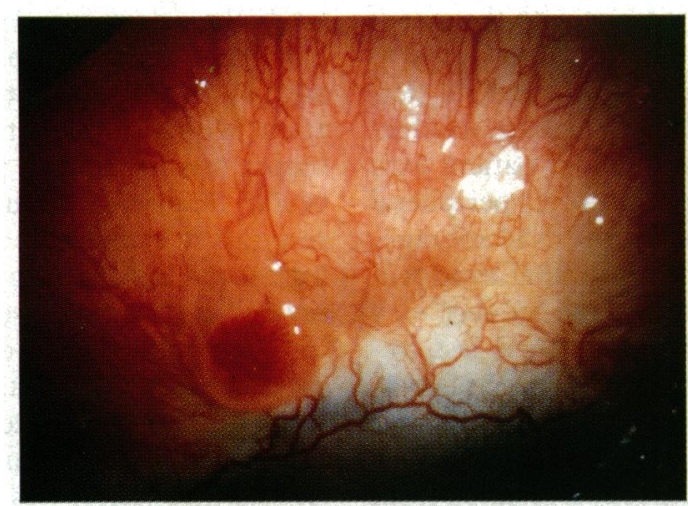

Figure $7 B$ The same patient five weeks later, showing excessive granulation tissue which developed during recovery. It was centred on a perforating deep scleral artery.

Plasma exchange was used in two patients with severe progressive renal disease: one had received pulsed immunosuppressive therapy, which had appeared to control the scleritis but not the renal disease; the other presented in severe renal failure and was also treated with oral cyclophosphamide and prednisolone.

Azathioprine was substituted for cyclophosphamide in one patient in remission, and prompted an immediate relapse.

Surgery was required in one patient. A corneoscleral patch graft was performed for a limbal perforation after the disease had been controlled medically (Fig 5C).

\section{CLINICAL PROGRESS}

In areas of conjunctival necrosis exposed sclera became covered by fibrovascular tissue from adjacent conjunctiva. These neovascular membranes contracted, distorting the surrounding conjunctival vascular pattern (Figs 7A, B, C). Granulation tissue was pronounced in one patient (Fig 7B). It arose in a zone of scleral necrosis, was centred on a perforating deep scleral artery, and later became infarcted.

During remission marginal corneal infiltrates became smaller, better defined, and more refractile in nature but did not disappear completely (Fig 7D).

FOLLOW-UP

Six out of 10 patients required no further pulsed immunosuppressive therapy to control their scleritis (follow-up 11 to 72 months, mean 25

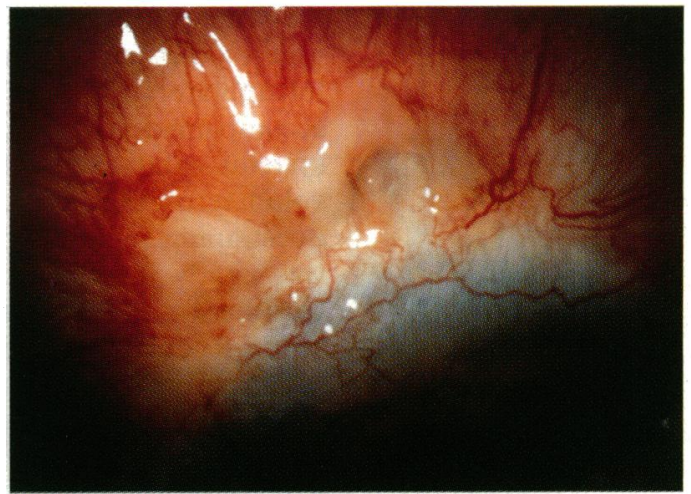

Figure 7C After involution of the granulation tissue, the exposed sclera became covered by a fibrovascular membrane. This contracted, distorting the conjunctival vascular pattern.

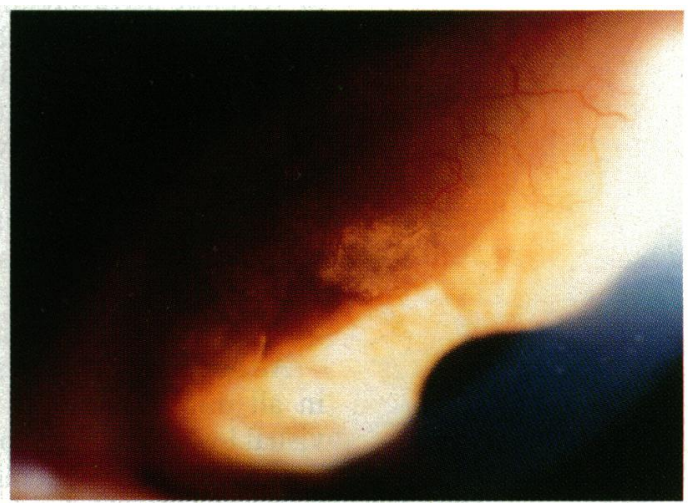

Figure 7D Corneal stromal infiltrates during remission, demonstrated by retroillumination in the same eye.

months). One (two cases) or two (two cases) further pulsed treatments were necessary in four patients.

Five patients had relapses of their systemic WG giving rise to acute renal failure (1), progressive renal failure (2), pericardial effusion (1), rashes (1), and arthralgia (3). One patient now requires haemodialysis.

Two out of 10 patients lost visual acuity in one eye after presentation: from 6/36 to counting fingers due to a macular hole secondary to cystoid macular oedema and uveitis in one; and from $6 / 24$ to $5 / 60$ due to retinal vascular occlusion complicating thrombocytosis in the other. Anterior segment changes such as guttering and perforation did not produce visual loss.

Intravenous methylprednisolone and cyclophosphamide were well tolerated apart from transient hyperglycaemia and electrolyte disturbances. Four patients became mildly cushingoid (with weight gain and facial changes), and one developed osteoporosis on prolonged systemic steroids. Alopecia was symptomatic in two patients. Pneumonia associated with cyclophosphamide-induced leucopenia and requiring admission to hospital arose in two patients. One patient suffered from herpes simplex virus pharyngitis. Later, when his WG was inactive, he died of a viral myocarditis. Although he was not severely leucopenic (leucocytes $3 \cdot 3 \times 10^{\%} / \mathrm{dl}$ ), this may have been related to his immunosuppression.

\section{Discussion}

This paper considers the manner in which WG 
may present in an eye clinic, though our cases reflect a bias in this centre towards corneoscleral inflammatory disease. The mildest scleritis was accompanied by critical involvement of other systems, and even when no symptoms were volunteered they could be revealed by careful questioning. The discovery of systemic disease was vitally important to all our patients, and treatment of this, rather than the eye in isolation, reduced ocular morbidity.

The scleral changes with which these patients presented included episcleral vasodilatation and oedema, scleral oedema, and scleral necrosis, which was often accompanied by destruction of the overlying conjunctiva. Although conjunctival necrosis is a dramatic physical sign, the presence of scleral necrosis correlated best with the extent of systemic involvement.

It has been proposed that corneoscleral destructive disease straddling the limbus characterises systemic vasculitis. ${ }^{2021}$ Every patient with scleral necrosis did have multifocal, peripheral corneal infiltrates beneath Bowman's membrane, showing the vulnerability of the limbal arcades in this condition. This is to be the subject of a further paper. Corneal guttering arose less frequently, and always adjacent to necrotic sclera.

Although scleritis was the presenting symptom in all our patients, it was not responsible for visual loss. When this occurred, it resulted from cystoid macular oedema complicating uveitis in one case and retinal vascular occlusion in another. The latter was associated with reactive thrombocytosis, highlighting the susceptibility of damaged vascular beds to alterations in blood viscosity.

Despite their ophthalmological presentation, our patients gave histories revealing systemic disease, ANCA levels were raised, and investigations confirmed involvement of respiratory tract and kidneys in all but two. Our inclusion of two subjects $(2,10)$ with scleritis and raised ANCA titres, but without the full clinical criteria of Fauci et $a l,{ }^{2}$ reflects our view that this investigation may enable diagnosis earlier in the natural history of the disease. Both patients already had widespread vasculitis requiring systemic treatment.

\section{CLINICAL ASSESSMENT}

Although a careful history, and systemic and otolaryngological examination, exposed systemic inflammatory disease in our patients, further investigations were helpful in confirming the diagnosis and systems affected, and monitoring response to treatment.

Even in the absence of symptoms we frequently found radiological evidence of sinusitis or pulmonary cavitation. Renal impairment, which was found at presentation in most of our patients, was also always asymptomatic. Half the patients had significant proteinuria, and creatinine clearance was reduced in seven out of 10 . Furthermore, renal disease was seen in patients with and without scleral necrosis: hence mild scleritis may be the presenting feature of incipient renal failure.

The activity of WG is reported to be better estimated by CRP than ESR, ${ }^{22}$ but both investi- gations proved equally sensitive during the assessment and management of our patients. Seven subjects presented with an ESR of over $80 \mathrm{~mm} / \mathrm{h}$, and two of the remainder were already on steroid therapy. In addition there was usually anaemia, leucocytosis, and thrombocytosis.

Immune complexes, complement, and serum immunoglobulins were raised in some patients, but, as in other studies, these were not consistent findings. ${ }^{23}$ One patient had a positive test for rheumatoid factor, but with the exception of ANCA other autoantibodies were not found in our patients.

The initiation of long term immunosuppressive therapy demands confident diagnosis of the underlying systemic disease. In this study the only diagnostic histology was obtained from nasal mucosal biopsy in one case after normal findings on otolaryngological examination. We consider this to be the most appropriate biopsy site in patients presenting with anterior ocular disease, even in the absence of upper respiratory symptoms. However, precise histological confirmation may still be difficult in both severe $^{18}$ and early disease, ${ }^{24}$ and inconclusive histology should never delay treatment.

As in previous reports ${ }^{818}$ the multifocal nature of this disease precluded the use of histology as a sole criterion for inclusion in our study. The ANCA titre (RIA) was increased in every case, usually at presentation but later in the course of the disease in two patients. Whenever it could be characterised by IIF, granular cytoplasmic staining was found, supporting the diagnosis of WG as opposed to other forms of vasculitis. The quantification of ANCA by RIA also provided a useful indicator of disease activity with which to monitor treatment.

\section{TREATMENT}

Oral steroids alone were insufficient to abort the progression of scleral necrosis. However, pulsed immunosuppressive therapy with intravenous methylprednisolone and cyclophosphamide successfully controlled scleritis whenever it was used. In the acute phase co-existing respiratory and renal disease had to be closely monitored, and two of our patients required plasma exchange because of progressive renal disease. Exacerbations were common, particularly following changes in treatment, but responded well to further pulsed immunosuppressive therapy.

Pulsed methylprednisolone and cyclophosphamide were well tolerated by all these patients. However, methylprednisolone therapy may be hazardous, and deaths have been reported..$^{25}$ The complications of prolonged oral prednisolone therapy are well known, and cyclophosphamide also has many side effects, including leucopenia, ${ }^{26}$ haemorrhagic cystitis, ${ }^{27}$ alopecia, ${ }^{28}$ teratogenesis, ${ }^{29}{ }^{30}$ and malignancy. ${ }^{31}$ Two of our patients developed profound cyclophosphamide-induced leucopenia, and in both cases this was followed by pneumonia.

We routinely check blood counts twice weekly during the inception of cyclophosphamide therapy, and monthly thereafter. However, the control of WG with immunosuppressive drugs may itself put a patient's life at risk, and many 
ophthalmologists will wish to collaborate with physicians experienced in lymphocytotoxic therapy.

Ophthalmologists must be able to recognise the patient whose eye disease is the presenting feature of a life-threatening systemic vasculitis. Previous studies have described inexorable progression of the ocular disease in WG, but with prompt diagnosis and treatment of the underlying systemic disease this need not occur.

The authors thank Dr C M Lockwood for his advice and assistance with ANCA analysis.

Paul Meyer is in receipt of a research fellowship from the Wellcome Trust.

1 Godman GC, Churg J. Wegener's Granulomatosis. Pathology and review of the literature. Arch Pathol 1954; 58: 533-53.

2 Fauci AS, Haynes BF, Katz P, Wolff SM. Wegener's granulomatosis: prospective clinical and therapeutic experience with 85 patients for 21 years. Ann Intern Med 1983; 98: 7685 .

3 Walton EW. Giant cell granuloma of the respiratory tract (Wegener's granulomatosis). Br Med F 1958; ii: 265-70.

4 Bullen CL, Liesegang TJ, McDonald TJ, DeRemee RA. Ocular complications of Wegener's granulomatosis. Ocular complications of W
Ophthalmology 1983; 90: 279-90.

5 Straatsma BR. Ocular manifestations of Wegener's Straatsma BR. Ocular manifestations of We
granulomatosis. Am $\mathcal{f} O$ phthalmol 1957; 44: 789-99.

6 Brady HR, Israel MR, Lewin WH. Wegener's granulomatosis and corneoscleral ulcer. FAMA 1965: 193: 248-9.

7 Austin P, Green WR, Sallyer DC, et al. Peripheral corneal degeneration and occlusive vasculitis in Wegener's granulomatosis. Am $\mathcal{F}$ Ophthalmol 1978; 85: 311-7.

8 Spalton DJ, Graham EM, Page NGR, Sanders MD. Ocular changes in limited forms of Wegener's granulomatosis. $\mathrm{Br} F$ Ophthalmol 1981; 65: 553-63.

9 Donald KJ, Edwards RL, McEvoy JDS. An ultrastructural study of the pathogenesis of tissue injury in limited Wegener's granulomatosis. Pathology 1976;8: 161-9.

10 Van der Woude FJ, Rasmussen N, Lobatto S, et al. Autoantibodies against neutrophils and monocytes; tool for diagnosis and marker for disease activity in Wegener's granulomatosis Lancet 1985; 425

11 Gross WL, Ludeman G, Kiefer G, Lehmann H. Anticytoplasmic antibodies in Wegener's granulomatosis. Lancet 1986; i: 806

12 Savage COS, Jones S, Winearls CG, Marshall PD, Lockwood CM. Prospective study of radioimmunoassay for antibodies against neutrophil cytoplasm in the diagnosis of systemic vasculitis. Lancet 1987; i: 1389-93.
13 Goldschmeding R, Huinink DTB, Faber N, et al. Identification of the ANCA antigen as a novel myeloid lysosomal serine protease. APMIS $1989 ; 97$ (suppl 6): 46 .

14 Falk RJ, Jennett JC. Anti-neutrophil cytoplasmic autoantibodies with specificity for myeloperoxidase in patients with systemic vasculitis and idiopathic necrotizing and crescentic glomerulonephritis. N Engl F Med 1988; 318: 1651-7.

15 Cohen Tervaert JW, Goldschmeding R, Elema JD, et al. Autoantibodies against myeloid lysosomal enzymes in crescentic glomerulonephritis. Kidney Int 1990; 37: 799806.

16 Hollander D, Manning RT. The use of alkylating agents in the treatment of Wegener's granulomatosis. Ann Intern Med 1967; 67: 393-8.

17 Harrison HL, Linshaw MA, Lindsley CB, Cuppage FE. Bolus corticosteroids for initial treatment of Wegener's granulocorticosteroids for initial treatment of
matosis. $7 A M A$ 1980; 244: 1599-600.

18 Pinching AJ, Lockwood CM, Pussell BA, et al. Wegener's granulomatosis: observations on 18 patients with severe renal disease. $Q$ f Med 1983; 52: 435-60.

19 Meyer PAR, Watson PG, Franks W, Dubord P. 'Pulsed' immunosuppressive therapy in the treatment of immunologically induced corneal and scleral disease. Eye 1987; 1: 487-95.

20 Watson PG, Booth Mason S. Fluorescein angiography in the differential diagnosis of sclerokeratitis. $\mathrm{Br} \mathcal{f}$ Ophthalmol 1987; 71: 145-51.

21 Watson PG. Vascular changes in peripheral corneal destructive disease. Eye 1990; 4: 65-73.

22 Hind CRK, Winearls CG, Lockwood CM, Rees AJ, Pepys MB. Objective monitoring of activity in Wegener's granulomatosis by measurement of serum C-reactive protein granulomatosis by measurement of serum C-reac
concentration. Clin Nephrol 1984; $21: 341-5$.

23 Shillitoe EJ, Lehner T, Lessoff MH, Harrison DFN. Immunological features of Wegener's granulomatosis. Lancet 1974; i: $281-4$.

24 McCluskey RT, Fienberg R. Vasculitis in primary vasculitides, granulomatoses and connective tissue diseases. Hum Pathol 1983; 14: 305-15.

25 Thompson JF, Chalmers DH, Wood RF, Kirkham SR, Morris PJ. Sudden death following high-dose intravenous methylprednisolone. Transplantation 1983; 36; 594-9.

26 Fauci AS, Wolff SM, Johnson JS. Effect of cyclophosphamide on the immune response in Wegener's granulomatosis. N Engl F Med 1971; 285; 1493-6.

27 Philips FS, Sterberg SS, Cronin AP, Vidol PM. Cyclophosphamide and urinary bladder toxicity. Cancer Res 1961; 21: 1577-89.

28 Anonymous. Drug causes of hair loss. Drug Ther Bull 1978; 16: 77 .

29 Toledo TM, Harper RC, Moser RH. Fetal effects during cyclophosphamide and irradiation therapy. Ann Intern Med 1971; 74: 87-91.

30 Greenberg LH, Verdes P, Tanaka KR. Congenital anomalies probably induced by cyclophosphamide. FAMA 1964; 188: 423-6

31 Marshall V. Premalignant and malignant skin tumours in immunosuppressed patients. Transplantation 1974; 17: $272-5$. 